\title{
Qualidade de vida: percepção de enfermeiros numa abordagem qualitativa
}

\section{Quality of life: nurses' perceptions under a qualitative approach}

\section{Calidad de vida: percepción de los enfermeros en una abordaje cualitativa}

\author{
Gisélia Alves Araújo', Maria Júlia Guimarães Oliveira Soares", Maria Emília Romero de Miranda Henriques"'I
}

1 Enfermeira. Mestre em Enfermagem de Saúde Pública. Enfermeira do Hospital Universitário Lauro Wanderley da Universidade Federal da Paraíba (UFPB). E-mail: ga.araujo@uol.com.br.

"Enfermeira. Doutora em Enfermagem. Docente do Departamento de Enfermagem da UFPB. E-mail: mmjulieg@yahoo.com.br.

III Enfermeira. Pós-Doutora pela Escuela de la Salud Publica de la Universidad de Granada, Espanha. Doutora em Enfermagem. Docente do Departamento de Enfermagem da UFPB. E-mail: milahenriques@hotmail.com.

\section{RESUMO}

Essa investigação teve como objetivos verificar o significado de qualidade de vida para os enfermeiros do Hospital Universitário Lauro Wanderley (HULW); verificar a percepção dos enfermeiros quanto a sua QV e identificar fatores que interferem na QV desses profissionais. O estudo do tipo exploratório-descritivo com enfoque qualitativo foi realizado no HULW de João Pessoa - PB. A amostra constituiu-se de 12 enfermeiros da área hospitalar. A coleta de dados ocorreu no mês de março de 2006, após aprovação do Comitê de Ética. Utilizou-se um questionário para a coleta dos dados e, para a análise desses dados, utilizou-se a técnica do Discurso do Sujeito Coletivo, que possibilitou a obtenção dos seguintes resultados para a formação das idéias centrais: um estado de harmonia, de vida equilibrada no âmbito bio-psico-sócio-espiritual; vida digna; péssima qualidade de vida pelo acúmulo de atividades; boa qualidade de vida, por poder conciliar várias coisas necessárias a essa qualidade; fator financeiro, excesso de trabalho, cobrança do mercado de trabalho interferindo na relação familiar; as dificuldades do cotidiano. Concluímos tornar-se necessário que, na atuação do enfermeiro, exista a preocupação de equilibrar as condições de trabalho e de vida pessoal em prol da satisfação de sua qualidade de vida.

Descritores: Qualidade de vida; Enfermagem; Pesquisa qualitativa.

\section{ABSTRACT}

This investigation aimed to verify the meaning of University Hospital Lauro Wanderley (HULW) nurses' quality of life; to verify nurses' perception in terms of quality of life and to identify factors that are interfering with those professionals' quality of life. This exploratory-descriptive study with a qualitative approach took place at the HULW, in J oão Pessoa, Paraíba/Brazil. The sample was composed of 12 hospital nurses. The data was collected during March of 2006, after being approved by the Ethics Committee. A questionnaire containing the subjects' identification and data related to their perception about quality of life was used, and for the data analysis the Discourse of the Collective Subject technique was used. That made possible the obtainment of the following results and the central ideas formation: a state of harmony, well-balanced life on bio-psychological-socio-spiritual aspects; self-respect; very bad quality of life due to accumulation of activities; good quality of life for being able to conciliate many necessary things for that matter; financial factors, work load, job market demands interfering in personal relationships; daily difficulties. It was possible to conclude that it is necessary, in the nurses' practice, to find a balance between working conditions and personal life, in order to have a better quality of life.

Descriptors: Quality of life; Nursing; Qualitative research.

\section{RESUMEN}

Esta investigación tuvo como objetivos verificar el significado de calidad de vida para los enfermeros del Hospital Universitario Lauro Wanderley (HULW); verificar la percepción de los enfermeros con relación a su CV e identificar factores que interfieren en la CV de esos profesionales. El estudio tipo exploratorio-descriptivo con enfoque cualitativo fue realizado en el HULW de João Pessoa - PB. La muestra se constituyó de 12 enfermeros de los sectores del área hospitalaria. La colecta de datos ocurrió en el mes de marzo del 2006, tras la aprobación del Comité de Ética. Fue utilizado un cuestionario para la colecta de los datos y, para el análisis de estos datos, se utilizó la técnica del Discurso del Sujeto Colectivo, que posibilitó la obtención de los siguientes resultados para la formación de las ideas centrales: un estado de armonía, de vida equilibrada en el ámbito Bio-psico-socio-espiritual; vida digna; pésima calidad de vida por la acumulación de actividades; buena calidad de vida, por poder conciliar varias cosas necesarias a esta calidad; factor financiero, exceso de trabajo, cobranza del mercado de trabajo interfiriendo en la relación familiar; las dificultades del cotidiano. Concluimos que se hace necesario que, en la actuación del enfermero, exista la preocupación de equilibrar las condiciones de trabajo y de vida personal en pro de la satisfacción de su calidad de vida.

Descriptores: Calidad de vida; Enfermería; Investigación cualitativa. 


\section{NTRODUÇÃO}

A evolução científica e tecnológica tem proporcionado ao ser humano conhecimento e instrumentos com diversas finalidades. Especialmente na área da saúde, a finalidade é capacitar os profissionais a prestarem uma assistência de qualidade. Inseridos nesse contexto, encontram-se os profissionais de enfermagem que têm procurado acompanhar todo esse avanço científico, ocupando todos os espaços de sua competência. Com isso, crescem os níveis de complexidade de atenção à saúde, tanto no âmbito ambulatorial como hospitalar, aumentando, consequentemente, a responsabilidade e o compromisso profissional.

Essa evolução tem provocado mudanças de ordem estrutural e organizacional no cotidiano das instituições de saúde e na vida daqueles que ali desempenham suas atividades profissionais. Essas mudanças têm sido uma tônica dos tempos modernos, ocorrendo de forma desordenada e abrupta, influenciando no estilo de vida das pessoas, podendo desencadear transtornos físicos e psicológicos.

As mudanças ocorridas na sociedade moderna têm sido excitantes, frenéticas e, muitas vezes, rápidas demais para que o homem possa absorver o seu ritmo e seu significado - o que as torna marcantes. Além disso, a competição excessiva, a busca constante pelo ter, a pressa, o medo do outro ser humano, a pressão diária que as pessoas bemsucedidas se impõem, inegavelmente afetam sua qualidade de vida. Desse modo, a qualidade de vida é prejudicada, pois uma vida de insegurança e incerteza deixa muito a desejar do que se almeja como uma vida de boa qualidade ${ }^{(1)}$

Assim, a expressão qualidade de vida (QV) passa a ser foco, nas últimas décadas, de estudos constantes, não apenas em termos teóricos, mas também na aplicabilidade em todos os campos da existência e da relação humana. Nessa visão, Dantas, Sawada e Malerbo ${ }^{(2)}$ compreendem QV como sendo "uma noção eminentemente humana e abrange muitos significados que refletem conhecimentos, experiências e valores de indivíduos e coletividades", inseridos no contexto histórico, social e cultural.

O termo QV tem sido bastante usado, mas nem sempre de forma abrangente. Para muitas pessoas, uma boa QV pode ser confundida com uma "vida boa", levando-se em consideração o aspecto material. A tendência atual é considerar QV sob um enfoque mais amplo, no qual se incluem: segurança, felicidade, lazer, saúde, condição financeira estável, amor e trabalho ${ }^{(3)}$. Na área da saúde, o interesse por QV decorre, em parte, do surgimento de novos paradigmas que passaram a influenciar as políticas e as práticas de saúde a partir das últimas décadas ${ }^{(4)}$.

O termo QV tem recebido definições diversificadas, das quais se destaca a que foi elaborada pelo grupo de especialistas da Organização Mundial da Saúde (WHOQOL Group) ${ }^{(5)}$ : “a percepção do indivíduo de sua posição na vida, no contexto da cultura e sistemas de valores nos quais ele vive e em relação aos seus objetivos, expectativas, padrões e preocupações". Embora este conceito tenha sido elaborado para todos os povos, conserva aspectos que vislumbram a individualidade e subjetividade de cada indivíduo inserido em sua cultura, através de uma avaliação pessoal.

Há ainda conceitos relacionados à saúde, também dentro de uma visão geral, mas que abordam aspectos mais ligados às enfermidades e/ou às intervenções em saúde. Seidl e Zannon ${ }^{(4)}$ referemse à qualidade de vida como um indicador apontado para um julgamento clínico de doenças específicas, e o impacto psicológico e social incidindo sobre as doenças de cunho físico.

Tecendo ainda considerações sobre a qualidade de vida, no âmbito da saúde, como atenção às necessidades humanas - materiais e espirituais destaca-se como ponto mais relevante a promoção da saúde. Esta leva-nos a refletir sobre o objeto saúde, que é um conceito em construção, dinâmico, que depende dos valores sociais, culturais e históricos, buscando uma relação harmoniosa para se viver com qualidade ${ }^{(6)}$. A nova concepção de saúde deixa de ser apenas ausência de "doença", mas refere-se ao bem-estar e qualidade da vida.

A qualidade de vida de um indivíduo pode ser alcançada a partir da obtenção da satisfação e realização pessoal, profissional, social etc. Assim, o trabalho é um elemento central para pensar a questão da QV, porque é por meio dele que o homem tem procurado satisfazer suas aspirações e alcançar sua qualidade de vida.

No âmbito hospitalar, o trabalho é desenvolvido num ambiente rico e heterogêneo, e se realiza por meio das várias áreas de atuação: das relações interpessoais entre os profissionais e a clientela assistida, ao cumprimento de tarefas consideradas árduas para os profissionais, inclusive os de enfermagem. Destaca-se também o ritmo de trabalho, por vezes acelerado, que pode resultar em pouco tempo disponível aos profissionais para relaxar, fazendo com que o trabalho torne-se penoso, capaz de causar desgaste físico e mental ${ }^{(7-8)}$.

Portanto, introduzir a discussão de QV no contexto hospitalar, permeando a qualidade do trabalho desenvolvido pela equipe de enfermagem, pressupõe considerar-se que boa parte da vida desses profissionais transcorre no seu local de trabalho. Para que esses profissionais obtenham satisfação e, consequentemente, qualidade de vida no trabalho, faz-se necessário que lhes sejam proporcionadas condições adequadas para o desempenho de suas atividades.

Dentro da equipe de enfermagem, o enfermeiro 
assume o papel de líder, de administrador das condições de vida no trabalho, sendo um facilitador para que se estabeleçam relações propícias de bemestar no convívio dentro da equipe. Portanto, tendo em vista o estilo de vida, destacam-se outras questões relacionadas à situação financeira, estrutura familiar, condições de manutenção de saúde e de moradia, meios de transporte, acesso ao lazer e ao desenvolvimento pessoal e profissional de modo contínuo e permanente.

As condições de trabalho às quais está submetida a equipe de enfermagem implicam em jornadas de trabalho que exigem a realização de uma multiplicidade de funções repetitivas, de ritmo excessivo, dentre outros aspectos. Tais condições podem gerar o absenteísmo no trabalho, repercutindo diretamente na produtividade e na qualidade de vida desses profissionais ${ }^{(9)}$.

Considerando-se a atualidade do tema qualidade de vida e sua abrangência, e vivenciando-se o cotidiano do trabalho do enfermeiro, emerge a importância deste estudo, como forma de contribuir para uma reflexão crítica desse profissional sobre sua qualidade de vida e a influência da mesma no seu trabalho. Uma vez que a essência do trabalho do enfermeiro está centrada no cuidado ao ser humano, contraditoriamente esse profissional poderá estar vivendo de forma descuidada, o que poderá repercutir na assistência prestada ao paciente.

Desse modo, alguns questionamentos surgiram: Qual o significado de qualidade de vida para os enfermeiros? Qual a percepção dos enfermeiros acerca de sua qualidade de vida? Quais os fatores que interferem na qualidade de vida dos enfermeiros? Nesse sentido, o presente estudo teve como objetivos: verificar o significado de qualidade de vida para os enfermeiros do Hospital Universitário Lauro Wanderley; verificar a percepção dos enfermeiros quanto a sua qualidade de vida e identificar os fatores que estão interferindo na qualidade de vida desses profissionais.

\section{METODOLOGIA}

Este estudo teve um caráter exploratóriodescritivo com um enfoque qualitativo. Foi realizado no Hospital Universitário Lauro Wanderley, situado na cidade de João Pessoa - PB, envolvendo 12 enfermeiros dos setores da área hospitalar, como: clínica médica (alas $A$ e B); área de doenças infectocontagiosas, pediátrica, obstétrica, cirúrgica (alas A e B); o Serviço de Pronto Atendimento (SPA); o centro cirúrgico e o Centro de Terapia Intensiva (CTI) adulto, pediátrico e neonatal.

Para viabilizar a coleta de dados, foi utilizado um instrumento que constou de duas partes: a primeira, com identificação dos sujeitos da pesquisa; a segunda, com dados relacionados ao significado e à percepção dos participantes acerca de sua qualidade de vida e aos fatores que interferem na mesma.

A coleta dos dados ocorreu no mês de março de 2006, após aprovação do Comitê de Ética em Pesquisa, do Hospital Universitário Lauro Wanderley da Universidade Federal da Paraíba, com protocolo de número 007/06. Os participantes do estudo assinaram o Termo de Consentimento Livre Esclarecido, de acordo com a Resolução 196/96 do Conselho Nacional de Saúde ${ }^{(10)}$.

Para a análise dos dados, foi utilizada a técnica do Discurso do Sujeito Coletivo, que envolve os seguintes passos operacionais: seleção das expressões-chave de cada discurso particular, as quais se constituem em segmentos contínuos ou descontínuos do discurso e revelam o principal do conteúdo discursivo; identificação da Idéia Central de cada uma dessas expressões-chave, constituindo a síntese do conteúdo destas expressões, ou seja, o que elas querem efetivamente expressar; identificação das Idéias Centrais semelhantes ou complementares e, por último, reunião das expressões-chave referentes às Idéias Centrais semelhantes ou complementares em um discurso síntese, que é o Discurso do Sujeito Coletivo ${ }^{(11)}$.

\section{RESULTADOS E DI SCUSSÃO}

\section{Caracterização dos sujeitos}

Este estudo foi realizado com 12 enfermeiros, com idades entre 25 e 55 anos. Destes, 5 (41,7\%) tinham idade entre 25 e 34 anos; 4 (33,3\%), entre 35 e 44 anos; e 3 (25\%), entre 44 e 55 anos. Esses dados revelam uma amostra constituída de indivíduos em idade potencialmente produtiva, conforme apontam dados de pesquisa do DATASUS, que considera produtivo o indivíduo que se encontra no segmento etário de 15 a 64 anos $^{(12)}$.

Considerando a época de realização do estudo, os achados permitiram inferir que, dos participantes, $10(83,3 \%)$ pertenciam ao sexo feminino e dois $(16,6 \%)$ ao sexo masculino. Desse modo, apesar de já se fazer notório o interesse do sexo masculino na Enfermagem, ainda prevalece nesta o sexo feminino - reforçando os achados do estudo que confirmam ainda a predominância desse sexo na profissão(13).

Quanto ao estado civil, $9(75,0 \%)$ dos pesquisados eram casados; e $3(25,0 \%)$, solteiros. Em relação ao número de filhos, $5(41,7 \%)$ dos participantes tinham dois filhos; 2 (16,7\%) tinham três filhos; $2(16,7 \%)$ tinham apenas 1 filho. Apenas $3(25 \%)$ dos profissionais não possuíam filhos. Em se tratando de renda familiar, $4(33,3 \%)$ dos sujeitos não responderam; $5(41,7 \%)$ informaram receber mais de dez salários mínimos e 3 (25\%) recebiam menos de dez salários mínimos. No que diz respeito à formação acadêmica, $9(75 \%)$ dos profissionais afirmaram possuir curso de especialização e 3 (25\%) possuíam graduação em Enfermagem. 
O tempo de formação profissional dos sujeitos do estudo variou de um ano a vinte anos: $4(33,2 \%)$ dos pesquisados possuíam tempo de formação profissional entre 6 e 10 anos; $2(16,7 \%)$ tinham de 1 a 5 anos ; 2 (16,7\%) tinham de 11 a 15 anos; 2 $(16,7 \%)$ de 16 a 20 anos e $2(16,7 \%)$ tinham mais de vinte anos. Quanto ao tempo de serviço na instituição pesquisada, constatou-se que $6 \quad(50,0 \%)$ dos participantes encontravam-se no serviço entre 1 a 5 anos. Para a questão relacionada a outro vínculo empregatício, $6(50,0 \%)$ profissionais, relataram possuir outro emprego e os outros $6 \quad(50,0 \%)$ responderam que não possuíam outro vínculo.

\section{A qualidade de vida na ótica dos enfermeiros}

Os quadros a seguir apresentam as questões norteadoras e as respectivas Idéias Centrais do discurso dos sujeitos participantes da pesquisa, agrupados de acordo com a técnica do Discurso do Sujeito Coletivo (DSC).

No Quadro 1 pode ser observado, no tocante à Idéia Central 1, o Discurso do Sujeito Coletivo dos enfermeiros, que expressa como significado de qualidade de vida o entrelaçamento, de forma harmônica, de vários elementos considerados essenciais à vida, o que lhe possibilita ter uma vida equilibrada, geradora de bem-estar no seu dia-a-dia.

Quadro1: I déias Centrais e Discurso do Sujeito Coletivo de enfermeiros do HULW em resposta à questão: $\mathbf{O}$ que significa qualidade de vida para você?

\begin{tabular}{|c|}
\hline I déia Central 1 \\
\hline Um estado de harmonia, de vida equilibrada no âmbito bio-psico-sócio-espiritual \\
\hline Discurso do Sujeito Coletivo \\
\hline $\begin{array}{l}\text { [...] Significa um estado de harmonia entre os diversos setores da vida, acredito na necessidade do indivíduo } \\
\text { ter um estado de vida equilibrada no âmbito bio-psico-social-espiritual para estar bem consigo mesmo, com } \\
\text { outros e gostando do que faz. [...] Significa um conjunto de fatores que fazem com que o ser humano tenha } \\
\text { um bem-estar no seu dia-a-dia quando fisicamente, economicamente e socialmente estamos bem. [...] É ter: } \\
\text { saúde, emprego, salário, moradia, família, lazer, educação e paz (Deus). }\end{array}$ \\
\hline I déia Central-2 \\
\hline Vida digna \\
\hline Discurso do Sujeito Coletivo \\
\hline $\begin{array}{c}\text { [...] Significa também uma vida digna para você poder aproveitar o que a vida oferece, podendo passear, } \\
\text { viajar, estudar, brincar, cantar [...]. Esses fatores levam o indivíduo a ter uma posição que pode gerar ou não } \\
\text { um grau de satisfação. }\end{array}$ \\
\hline
\end{tabular}

Apesar de haver uma variedade de conceitos em relação à QV, é importante ressaltar que aspectos fundamentais deste constructo - como subjetividade, multidimensionalidade e dimensões positivas e negativas - devem ser considerados ${ }^{(4)}$.

Qualidade de vida é um tema que tem merecido uma abordagem dentro de uma visão ampliada, de modo a considerar os aspectos fundamentais de sua subjetividade. Para Gonçalves e Vilarta(14), QV significa muitas coisas necessárias no cotidiano para a sobrevivência incluindo transporte, habitação, lazer, trabalho entre outras necessidades inerentes ao ser humano, levando-se em consideração que cada ser tem sua singularidade e especificidade.

A Idéia Central 2, do Quadro 1, apresenta o DSC, ressaltando a importância de se ter uma "vida digna", compreendida como fator essencial para proporcionar QV, na perspectiva da diversão e do lazer - entendidos como elementos indispensáveis que Ihes permitem "aproveitar a vida". Nessa perspectiva, essas necessidades podem contribuir para que a pessoa viva com maior satisfação e, possivelmente, se fortalecendo para o enfrentamento do dia-a-dia.

O conceito de qualidade de vida incorpora não só bens materiais, mas outras dimensões são necessárias para que o indivíduo alcance uma vida digna. Portanto a qualidade de vida diz respeito a todos os aspectos, que permeiam o vivenciar do ser humano.

Diante da subjetividade e abrangência de requisitos necessários para uma boa qualidade de vida, faz-se necessário considerar o homem em sua totalidade - um ser bio-psico-sócio-espiritual. A não contemplação de um desses requisitos pode interferir decisivamente na qualidade de vida deste, adotando assim uma postura reducionista.

Para Paschoal(15) as avaliações subjetivas são limitadas, podendo ser instáveis, sujeitas às variações do cotidiano. Portanto, o que hoje é considerado como uma boa $\mathrm{QV}$, pode não ser mais daqui a algum tempo, ou mudar repentinamente, dependendo do estado de espírito ou até mesmo do humor.

De acordo com a Idéia Central 1, descrita no Quadro 2, o DSC demonstra que o acúmulo de atividades impõe um ritmo que tem provocado quebra na harmonia entre os vários fatores da vida, onde o econômico tem se constituído o mais marcante na busca de satisfazer as necessidades consideradas básicas; com base no DSC, a qualidade de vida foi percebida com má qualidade, ou seja, "péssima". 
Quadro 2: Idéias centrais e Discurso do Sujeito Coletivo de enfermeiros do HULW em resposta à questão: Como você percebe sua qualidade de vida?

I déia Central-1

Péssima qualidade de vida pelo acúmulo de atividades Discurso do Sujeito Coletivo

No momento está péssima, exatamente pelo acúmulo de atividades, o que não me permite aproveitar a casa, a família, o lazer. Sinto que, no momento, a vida como um todo não se encontra em harmonia, porque uma área

ou outra tem sido negligenciada [...] A busca pela qualidade de vida, hoje, nos remete muitas vezes à dependência econômica, nos fazendo escravos do trabalho. O ritmo de vida nos impõe a correr mais, não para conseguir mais coisas, mas para mantê-las.

\begin{tabular}{|c|}
\hline I déia Central-2 \\
\hline $\begin{array}{l}\text { I déia Central-2 } \\
\text { Boa por poder conciliar várias coisas necessárias à qualidade de vida }\end{array}$ \\
\hline $\begin{array}{l}\text { Discurso do Sujeito Coletivo } \\
\end{array}$ \\
\hline $\begin{array}{c}\text { Acho que minha vida tem uma boa qualidade, pois tive oportunidade de ter uma profissão que gosto [...], que } \\
\text { tenho vocação, tenho uma boa harmonia familiar, lazer, viajar, ir ao cinema, e tempo para conversar com } \\
\text { amigos, pessoas queridas e tudo isso me faz bem. Digo que tenho direito a todos os itens que classifico como } \\
\text { uma boa qualidade de vida. }\end{array}$ \\
\hline
\end{tabular}

A qualidade de vida do ser humano depende da satisfação de várias necessidades inerentes ao ser humano. Desse modo, torna-se inegável que a questão da QV está inserida na luta diária pelas condições de sobrevivência econômica das pessoas. Assim, uma simples observação revela a precariedade das condições de trabalho que influencia, não só no aumento da lucratividade do sistema econômico como um todo, como também na perspectiva de piora da QV das pessoas ${ }^{(16)}$.

Nesse sentido, verifica-se que na atualidade é comum as pessoas afirmarem que se torna mais fácil "falar de qualidade de vida do que viver uma vida com qualidade". Se por um lado a tecnologia proporcionou oportunidades de bem-estar do indivíduo, por outro lado criou ameaças à vivência e suas relações, bem como a transcendência humana ${ }^{(17)}$.

A segunda Idéia do Quadro 2 revela um Discurso do Sujeito Coletivo de forma positiva, haja vista que os componentes da vida apresentados estão em sintonia, envolvendo desde a vocação profissional quando os sujeitos expressam a satisfação da profissão que exercem - até o direito de desfrutar momentos prazerosos em família. O DSC possibilita evidenciar que os participantes percebem como boa sua qualidade de vida, dando um sentido de felicidade.

A busca pela felicidade faz parte da natureza humana e é tão antiga quanto sua existência. Porém, para que uma pessoa se sinta feliz, faz-se necessária a satisfação de várias necessidades, como ter saúde, estar de bem consigo mesmo e com o seu trabalho, podendo tudo isso ser considerado como qualidade de $v{ }^{(18)}$. Vale salientar que os elementos que podem agir no sentido de maior ou menor grau de satisfação dependem do valor que cada pessoa dá aos elementos elegidos como importantes para sua qualidade de vida. Acreditando nesse fato, podemos afirmar que ter qualidade de vida não é necessariamente ter esses elementos, mas acreditar e valorizar cada um dentro do que se acredita como essencial para viver melhor.

Desta forma, os seres humanos podem ser considerados iguais quanto à espécie, porém distintos quanto à maneira de viver a vida, tendo em vista seus objetivos, anseios de realizações, valores e crenças, dentre outros. Tudo isto faz com que a avaliação da qualidade de vida referida por cada pessoa possa sofrer modificação, mediante as mudanças impostas pela vida. Assim, em resposta a alterações importantes ocorridas na vida das pessoas, elas também mudam, não apenas a avaliação das coisas, mas a forma pela qual avaliam sua qualidade de vida ${ }^{(15)}$.

O Quadro 3 destaca o Discurso do Sujeito Coletivo referente à primeira Idéia Central, onde os pesquisados enumeram as dificuldades do cotidiano, dentre as quais o fator financeiro destaca-se como principal elemento que tem interferido na qualidade de vida dos mesmos. $\mathrm{Na}$ atualidade, devido à defasagem salarial imposta pela falta de uma política justa que garanta ao cidadão não só a sobrevivência, mas uma vida digna tem surgido a necessidade de se ter mais de uma fonte de renda que atenda às necessidades básicas do cidadão; dessa forma, a solução mais viável tem sido o pluriemprego. 
Quadro 3: Idéias Centrais e Discurso do Sujeito Coletivo de enfermeiros do HULW em resposta à questão: Quais os fatores que interferem na sua qualidade de vida? I déia Central-1

\begin{tabular}{|c|}
\hline I déia Central-1 \\
\hline $\begin{array}{l}\text { Fator financeiro, excesso de trabalho, cobrança do mercado de trabalho interferindo na relação } \\
\text { familiar. }\end{array}$ \\
\hline Discurso do Sujeito Coletivo \\
\hline $\begin{array}{c}\text { O principal fator que interfere na minha qualidade de vida é a renda familiar, o fator financeiro, ter que } \\
\text { trabalhar em mais de um serviço; carga horária excessiva de trabalho em decorrência de baixos salários, para } \\
\text { se aproximar de um salário adequado às despesas necessárias, de forma a poder conseguir sobreviver. E com } \\
\text { isso o tempo para as outras coisas torna-se mais curto [...]. Creio que o acúmulo de atividades ligadas à área } \\
\text { profissional, a cobrança do meio acadêmico e do mercado de trabalho, que exige de nós a busca pelas } \\
\text { "especializações", tem interferido na relação familiar, principalmente no tempo disponível para as atividades } \\
\text { como o lazer em família. }\end{array}$ \\
\hline I déia Central-2 \\
\hline As dificuldades do cotidiano \\
\hline Discurso do Sujeito Coletivo \\
\hline $\begin{array}{c}\text { O corre-corre do dia-a-dia para dar uma melhor qualidade de vida aos filhos [...], o estresse constante no } \\
\text { trabalho e o trânsito intenso também são fatores que tiram minha harmonia de vida [...], trabalhar em um } \\
\text { serviço desarmonioso ou desorganizado, falta de satisfação no trabalho, dormir pouco, pouco lazer, pouca } \\
\text { oração, excesso de responsabilidades, estresse e ausência de casa. [...] falta de exercícios físicos, alimentação } \\
\text { inadequada, [...] saúde e bem-estar. }\end{array}$ \\
\hline
\end{tabular}

O acúmulo de vínculos empregatícios tem provocado excesso de horas trabalhadas e, consequentemente, o tempo para as demais áreas da vida acaba por ficar sempre em segundo plano. Em contrapartida, o mercado de trabalho tem se tornado mais exigente mediante o avanço tecnológico, requerendo um profissional mais qualificado, ou seja, mais especializado. Possivelmente, esses fatores têm prejudicado não só a vida pessoal, mas também a interação familiar.

Desse modo, o aumento da jornada de trabalho e horas extras, utilizado como recurso para minimizar as questões de ordem econômica, "rouba-lhe o tempo livre que deveria estar à disposição do descanso necessário para recuperação das energias físicas, para o lazer, para a convivência familiar de forma mais harmônica e menos conflituosa"(19).

A segunda Idéia Central do Quadro 3 mostra o Discurso do Sujeito Coletivo que retrata as dificuldades enfrentadas pelos participantes do estudo no cotidiano, através de suas referências a problemas como o estresse diário, envolvendo o excesso de trabalho, problemas com o trânsito e relacionamentos conflituosos. Esse DSC reflete a crise que a sociedade moderna vivencia em decorrência do desenvolvimento urbano, com o aumento populacional, impondo uma competitividade geradora de um estilo de vida prejudicial à saúde física e mental do indivíduo.

Nesse sentido, a crise da sociedade moderna tem gerado aumento da tristeza, da angústia e da solidão, apesar da expansão da indústria do lazer (jogos, internet, televisão, estilos musicais) e do aumento populacional. As pessoas estão sós no meio da multidão; fato esse decorre da falta de diálogo entre os grupos, em especial a família, que passou a caracterizar-se apenas como um grupo de pessoas que moram sob o mesmo teto. Em conseqüência, observa-se a deterioração da própria qualidade de vida da população que, mesmo contando com o avanço da medicina, da psicologia e da psiquiatria, apresenta um comportamento ansioso e estressado ${ }^{(20)}$.

\section{CONCLUSÃO}

Abordar o tema qualidade de vida é algo desafiador, pois a cada dia que passa a busca por um consenso acerca do conceito referente a esse tema parece levantar ainda mais questionamentos, que exigem reflexões amplas e profundas para os estudiosos da área. É também importante, uma vez que possibilita aos pesquisadores repensarem a qualidade dada a sua própria vida.

Este estudo permitiu observar que o significado de qualidade de vida para os entrevistados foi visto holisticamente, onde a harmonia na vida pessoal e familiar foi ressaltada; e reducionista, quando a QV foi compreendida como "vida digna" apenas no sentido de proporcionar os meios para aproveitar a vida, referindo-se às atividades de lazer.

No tocante à percepção da QV, foi mencionado, de forma positiva, considerada boa, por poderem articular o trabalho, a família, o financeiro, o lazer, dentre outros; e, de forma negativa, por estarem esses elementos em desequilíbrio devido às dificuldades financeiras, o excesso de trabalho, o pouco contato com a família e a falta de lazer.

Em relação aos fatores que interferem na QV, o principal fator identificado foi o financeiro, considerado forte elemento na estruturação familiar, tanto para a realização das necessidades e desejos pessoais, como para galgar o aperfeiçoamento e 
qualificação profissional. Além de outras dificuldades vivenciadas no cotidiano foram relatadas pelos participantes como: o acúmulo de atividades e responsabilidades, tornando a vida agitada e estressada, interferindo no sono, em uma alimentação inadequada, na falta de exercícios físicos, prejudicando a QV nos aspectos bio-psicosocio-espiritual.

Logo, torna-se necessário que na atuação do enfermeiro esteja presente a preocupação de valorizar o seu trabalho, mas também de buscar alternativas para equilibrar as condições de trabalho e de vida, em uma convivência harmoniosa que the permita sentir satisfação com a sua qualidade de vida.

\section{REFERÊNCI AS}

1. Lipp MEN. Estresse emocional: a contribuição de estressores internos e externos. Rev. psiquiatr. clín. 2001; 28(6): 347-9.

2. Dantas RAS, Sawada NO, Malerbo MB. Pesquisa sobre qualidade de vida: revisão da produção cientifica das universidades públicas do Estado de São Paulo. Rev Latino-am Enfermagem. 2003; 11(4): 532-8.

3. Vilarta R, Gonçalves A. Qualidade de vida concepções básicas voltadas à saúde. In: Gonçalves A, Vilarta R, editors. Qualidade de vida e atividade física: explorando teoria e prática. Barueri: Manole; 2004. p.27-62.

4. Seidl EMF, Zannon CMLC. Qualidade de vida e saúde: aspectos conceituais e metodológicos. Cad. Saúde Pública. 2004; 20(2): 580-8.

5. The Word Health Organization Quality of Life assessment (WHOQOL): position paper from the World Health Organization. Soc Sci Med. 1995; 41(10): 1403-9.

6. Ministério da Saúde. Política Nacional de Promoção da Saúde. Brasília (Brasil): Ministério da Saúde; 2006.

7. Mochel EG, Santos MH. O ambiente laboral dos profissionais de enfermagem em uma unidade neonatal. Revista Técnico-científica de Enfermagem. 2006; 4(15): 145-50.

8. Calderero ARL, Miasso Al, Corradi-Webster CM. Estresse e estratégias de enfrentamento em uma equipe de enfermagem de Pronto Atendimento. Rev. Eletr. Enf. [Internet]. 2008 [cited 2009 jul 20];10(1)51-62. Available from: http://www.fen.ufg.br/revista/v10/n1/v10n1a05.htm 9. Delgado LM, Oliveira BRG. Perfil epidemiológico do adoecimento dos profissionais de enfermagem de um hospital universitário. Revista Nursing. 2005; 87(8): 365-70.

10. Ministério da Saúde; Conselho Nacional de Saúde. Resolução № 196/96 - Normas regulamentadoras de pesquisa envolvendo seres humanos. Brasília (Brasil): Ministério da Saúde;
1996.

11. Lefèvre $F$, Lefèvre AMC. O discurso do sujeito coletivo: um novo enfoque em pesquisas qualitativas (desdobramentos). Caxias do Sul: EDUSC; 2005.

12. DATASUS [Internet]. Brasília: Ministério da Saúde (BR) [cited 2009 jul 30]. Indicadores demográficos. Razão de dependência. Available from: http://tabnet. datasus.gov.br/cgi/idb2002/a16.htm.

13. Erzinger AR, Trentini M. Enfermeiras e enfermeiros frente aos desafios no inicio da carreira profissional. Revista Técnico-Científica de Enfermagem. 2003; 1(5): 332-9.

14. Gonçalves A, Vilarta R. Qualidade de vida: identidades e indicadores. In: Gonçalves A, Vilarta R, org. Qualidade de vida e atividade física: explorando teoria e prática. Barueri: Manole; 2004. p. 3-25.

15. Paschoal SMP. Qualidade de vida do idoso: construção de um instrumento de avaliação através do método do impacto clínico [thesis]. São Paulo: Faculdade de Medicina/USP; 2004. 227p.

16. Gutierrez GL, Aspectos organizacionais e lúdicos da qualidade de vida: abordagem multidisciplinar. In: Gonçalves A, Vilarta R, org. Qualidade de vida e atividade física: explorando teoria e prática. Barueri: Manole; 2004. p.165-83.

17. Bramante AC. Qualidade de vida e lazer. In: Gonçalves A, Vilarta R, org. Qualidade de vida e atividade física: explorando teoria e prática. Barueri: Manole; 2004. p. 185-205.

18. Conte AL. Qualidade de vida no trabalho [Internet]. Santa Catarina: Revista FAE BUSINESS; 2004 Sep 16 [cited 2009 jul 30]. Available from: http://www.fae.edu/publicacoes/pdf/revista_fae_busi ness/n7/rev_fae_business_07_2003_gestao_10.pdf. 19. Veras VSD. Aumento da jornada: qual a repercussão na vida dos trabalhadores da enfermagem? [dissertation]. Natal: Faculdade de Enfermagem/UFRN; 2003. $121 \mathrm{p}$.

20. Cury A. 12 Semanas para mudar uma vida. Colinas: Editora Academia de Inteligência; 2004.

Artigo recebido em 20.05.08.

Aprovado para publicação em 06.05.09. Artigo publicado em 30.09.09. 